\title{
Biosynthesis of the $\alpha$-nitro-containing cyclic tripeptide psychrophilin
}

\author{
Muxun Zhao' ${ }^{1}$, Hsiao-Ching $\operatorname{Lin}^{1,2}$ and Yi Tang ${ }^{1,3}$ \\ The Journal of Antibiotics (2016) 69, 571-573; doi:10.1038/ja.2016.33; published online 9 March 2016
}

Psychrophilins A-H (1-8) are cyclic tripeptides isolated from different Penicillium species (Figure 1). ${ }^{1-4}$ These natural products are synthesized from one molecule each of L-tryptophan, the unnatural amino acid anthranilate (Ant) and an additional natural L-amino acid (proline: 1, 5-8; valine: 2; alanine: 3; and leucine: 4 ) through the actions of nonribosomal peptide synthetases (NRPSs). For example, psychrophilin B 2 is expected to be cyclized from the $\mathrm{L}-\operatorname{Trp}-\mathrm{L}-\mathrm{Val}-$ Ant tripeptidyl thioester attached to the last thiolation domain of the corresponding NRPS. Ant containing fungal peptidyl alkaloids have been well-characterized recently, most of which are structurally complex, multicyclic compounds. ${ }^{5}$ Generation of the 13-memberd macrocycle of psychrophilin is however highly unusual, highlighted by formation of the amide bond between the carboxylate of Ant and indole nitrogen of L-Trp, instead of the expected $\alpha-\mathrm{NH}_{2}$ of L-Trp. Interestingly in 1-4, the $\alpha-\mathrm{NH}_{2}$ of $\mathrm{L}$-Trp is oxidized into the rarely observed nitro group; ${ }^{1-3}$ whereas in 5-8, the same amino group is acetylated and further methylated. ${ }^{4}$

The timing of the L-Trp $\alpha-\mathrm{NH}_{2}$ modification (oxidation or acetylation/methylation) relative to macrocyclization is therefore an interesting biosynthetic question (Figure 2). The $\alpha-\mathrm{NH}_{2}$ group is significantly more reactive in the macrocyclization reaction compared with the indole nitrogen. Nucleophilic cyclization through the $\alpha-\mathrm{NH}_{2}$ (path c) is the canonical NRPS macrocyclization reaction and will lead to formation of a 10-membered macrolactam 11 that may undergo spontaneous quinazoline formation (Figure 2), as observed in other Ant and L-Trp containing tripeptide fungal NRPs such as in fumiquinazoline $\mathrm{F}^{6,7}$ Hence we hypothesize the $\alpha$-amino group of L-Trp may be oxidized to the nitro group prior to cyclization to prevent formation of 11, and allowing the indole nitrogen to serve as the nucleophile (path b). Alternatively, participation of the indole nitrogen in the cyclization reaction may be entirely controlled by the cyclization domain of the NRPS, and oxidation of the $\alpha-\mathrm{NH}_{2}$ occurs following formation of the cyclic tripeptide 9 (path $a$ ).

To investigate the biosynthesis of 2 and 3 , we sequenced the genome of the producing psychrotoleratnt fungus Penicillium rivulum (IBT 24420) using Illumina HiSeq. ${ }^{1}$ The $\sim 36 \mathrm{Mb}$ of genome was assembled and was scanned for NRPS genes using AntiSMASH. ${ }^{8} \mathrm{~A}$ total of 30 NRPS-encoding genes were found, ranging in sizes between mono-module and six-modules. Using the amino acid specificity code determined for adenylation domains that activate Ant, ${ }^{9}$ seven distinct modules were found and further analyzed for the potential psy cluster. Two candidate clusters encoded in scaffold 46 and scaffold 182 were found to be the most likely. The cluster encoded in scaffold 182 contains a trimodular NRPS (Pv182-1) and a cytochrome P450 monooxygenase (P450, Pv182-2). While the NRPS and P450 are the minimally required enzymes expected to produce 2 and 3 , the Ant-specific A domain was found to reside in the first module of the NRPS, hence inconsistent with the predicted order of amino acid activation (1: L-Trp; 2: L-Val/L-Ala; 3: Ant). Indeed, genetic inactivation of this cluster did not result in the abolishment of psychrophilin production (Supplementary Figure S6).

Two separate NRPS-encoding genes are found clustered in scaffold 46 (Figure 2 and Supplementary Table S2), including a dimodule NRPS Pv46-49 with the domain organization of A-PCP-C-A-PCP-C (domain abbreviations: A: adenylation; PCP: peptidyl-carrier protein; C: condensation) and a monomodule NRPS Pv46-51 with the domain organization of C-A-PCP- $\mathrm{C}_{\mathrm{T}}$, where the A domain is specific for Ant and the terminal $\mathrm{C}_{\mathrm{T}}$ is a putative cyclization domain also found in other macrocyclizing fungal NRPSs. ${ }^{7}$ A gene sandwiched between those encoding the NRPSs encodes a P450 enzyme Pv46-50 that shows the highest sequence identity $(\sim 40 \%)$ to isotrichodermin C-15 hydroxylase in trichothecene biosynthetic pathways. ${ }^{10}$ While the organization of the modules between the two NRPSs is consistent with that required for 2 biosynthesis, the division of NRPS modules into separate genes is rarely observed among fungal NRPSs. RT-PCR analysis showed that all three genes were actively transcribed under psychrophilin producing conditions (Supplementary Figure S5).

To establish if these genes constitute the psy cluster (GenBank accession: KU695586), we generated single-gene knockout of Pv46-49 (psyA), Pv46-51 (psyB) and Pv46-50 (psyC) using homologous recombination and analyzed the change in metabolite levels of the resulting $P$. rivulum strains (Supplementary Figure S2-S4). Deletion of each gene resulted in the complete abolishment of the production of $\mathbf{2}$

\footnotetext{
${ }^{1}$ Department of Chemical and Biomolecular Engineering, University of California, CA, USA; ${ }^{2}$ nstitute of Biological Chemistry, Academia Sinica, Taipei, Taiwan and ${ }^{3}$ Department of Chemistry and Biochemistry; University of California, CA, USA

Correspondence: Professor Yi Tang, Department of Chemical and Biomolecular Engineering, University of California, 5531 Boelter Hall, 420 Westwood Plaza, Los Angeles, CA 90095, USA.

E-mail: yitang@ucla.edu

Received 12 January 2016; revised 10 February 2016; accepted 12 February 2016; published online 9 March 2016
} 
<smiles>O=C1Nc2ccccc2C(=O)n2cc(c3ccccc32)CC([N+](=O)[O-])C(=O)N2CCC[C@H]12</smiles><smiles>[R]C1NC(=O)C([N+](=O)[O-])Cc2cn(c3ccccc23)C(=O)c2ccccc2NC1=O</smiles><smiles>[R3]c1cccc2c1c1cn2C(=O)c2ccccc2NC(=O)[C@@H]2CCCN2C(=O)[C@H](N([R])C(C)=O)[C@H]1[R]</smiles>

$2 \mathrm{R}=\mathrm{CH}\left(\mathrm{CH}_{3}\right)_{2}$ $3 \mathrm{R}=\mathrm{CH}_{3}$ $4 \mathrm{R}=\mathrm{CHCH}_{2}\left(\mathrm{CH}_{3}\right)_{2}$

$5 \mathrm{R}_{1}=\mathrm{R}_{2}=\mathrm{R}_{3}=\mathrm{H}$

$6 \mathrm{R}_{1}=\mathrm{CH}_{3}, \mathrm{R}_{2}=\mathrm{R}_{3}=\mathrm{H}$

$7 \mathrm{R}_{1}=\mathrm{CH}_{3}, \mathrm{R}_{2}=\mathrm{OH}, \mathrm{R}_{3}=\mathrm{H}$<smiles>CC(C)[C@H]1NC(=O)[C@@H](N)Cc2cn(c3ccccc23)C(=O)c2ccccc2NC1=O</smiles>

$8 \mathrm{R}_{1}=\mathrm{CH}_{3}, \mathrm{R}_{2}=\mathrm{R}_{3}=\mathrm{OH}$<smiles>O=C(N[C@@H](Cc1ccccc1)C(=O)N[C@H](COC(=O)c1ccccc1)Cc1ccccc1)c1ccccc1</smiles>

10

Figure 1 Compounds described in this work. Previously isolated psychrophilin A (1), B (2), C (3), D (4), E (5), F (6), G (7) and H (8). Psychrophilin I (9) is isolated in this work as an intermediate to 2. Dipeptide 10 was isolated from $\triangle p s y A$ knockout strain.

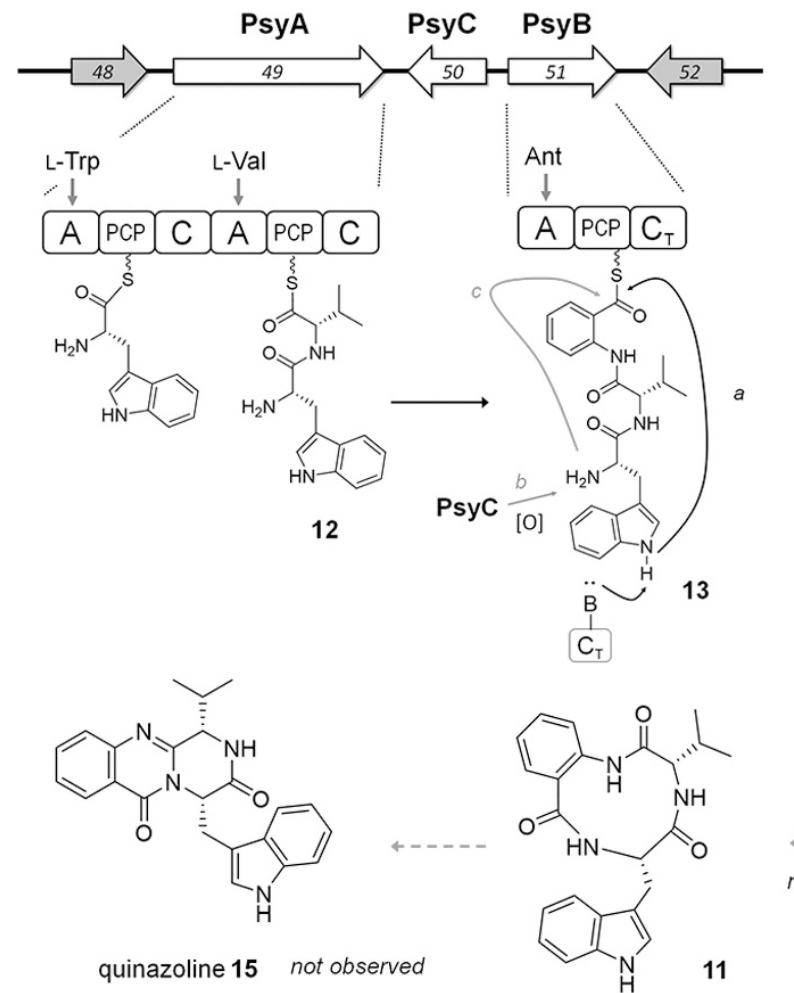

and 3, thereby confirming the involvement of the encoded enzymes in biosynthesis of psychrophilins (Supplementary Figure S6). In the NRPS knockout strains $\triangle p s y A$ and $\triangle p s y B$, no detectable intermediates related to 2 or 3 can be isolated. In the P450 deletion strain $\Delta p s y C$, a new metabolite with molecular weight of 404 and significantly earlier retention time on $\mathrm{C} 18$ reverse phase column emerged as a major product. The mass corresponds to the cyclized product of L-Trp-LVal-Ant, indicating this is indeed a related metabolite. Selected ionmonitoring using $m / z=405(\mathrm{M}+\mathrm{H})^{+}$showed that this compound is also present in the wild type strain, albeit only at trace quantities. The metabolite was purified from the mutant strain and fully characterized by NMR spectroscopy (Supplementary Table S4). The ${ }^{1} \mathrm{H}$ NMR data showed the characteristic signals of one isopropyl group $\left[\begin{array}{ll}\delta & 1.79\end{array}\right.$ $(\mathrm{m}, \mathrm{H}-21), \delta 0.84(\mathrm{~d}, J=6.5 \mathrm{~Hz}, \mathrm{H}-22)$ and $\delta 0.82(\mathrm{~d}, J=6.5 \mathrm{~Hz}$, $\mathrm{H}-23)$ ], two sets of signals of one indole group and one 1,2disubstituted benzyl group (Supplementary Table S4) for the side chains of L-valine, L-tryptophan and Ant, respectively. The upfield shift ( $\delta 3.75$ ) of $\mathrm{H}-2$ suggested that the $\alpha$-carbon in tryptophan was substituted by an amine group rather than a nitro group by comparing to that of $\mathbf{2}$ (Supplementary Table S3). The HMBC spectrum showed a key correlation of $\operatorname{Trp} \mathrm{H}-11$ ( $\delta$ 6.91) to Ant C-12 ( $\delta$ 169.0), confirming the linkage between Trp and Ant through an indole nitrogen. Therefore, the structure was established as a new compound psychrophilin I (9), not the 10-membered macrolactam 11. To confirm that $\mathbf{9}$ is the penultimate intermediate in the biosynthesis of 2, we supplied 9 to the blocked mutant of $\Delta p s y B$ at a final concentration of $0.2 \mathrm{mM}$. Following two days of culturing, restored production of 2 was observed, however the production of 3 remains abolished as expected (Supplementary Figure S6).

Based on the genetic inactivation and chemical complementation studies, we propose the biosynthesis of 2 as shown in Figure 2. The dimodular NRPS PsyA incorporates L-Trp and L-Val to yield the

P. rivulum genome Scaffold 46 (Pv46)

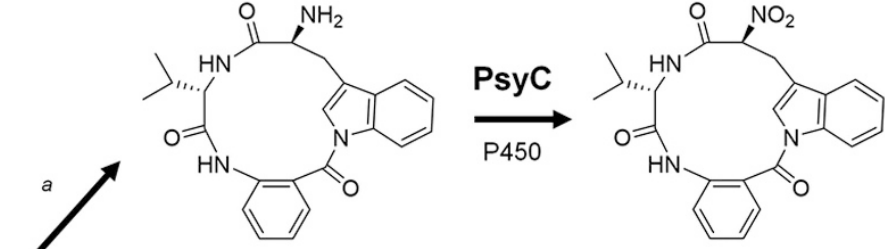

psychrophilin I (9)

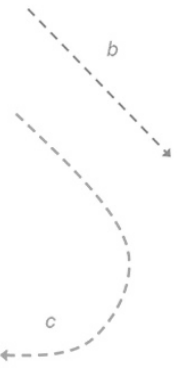

not observed

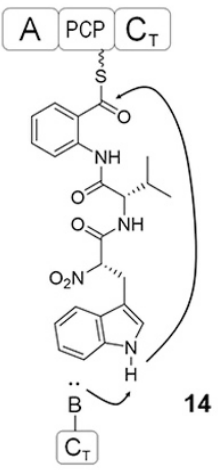

psychrophilin B (2)

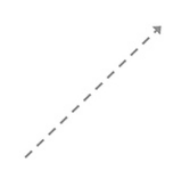

Figure 2 Biosynthesis of psychrophilin B and proposed biosynthetic pathway. A full color version of this figure is available at The Journal of Antibiotics journal online. 
L-Trp-L-Val dipeptidyl thioester 12. The monomodular NRPS PsyB activates Ant, which is condensed with $\mathbf{1 2}$ through the action of the terminal $\mathrm{C}$ domain in PsyA to yield the tripeptidyl thioester 13. The $\mathrm{C}_{\mathrm{T}}$ domain of PsyB is highly unusual in utilizing the indole nitrogen in the nucleophilic attack of the thioester to release the product 9 (path $a$ ). This reaction is mechanistically similar to that catalyzed by the monomodular NRPS Af12050 or TqaB in the fumiquinazoline A biosynthetic pathways, in which the indole nitrogen of fumiquinazoline $\mathrm{F}$ is acylated with $\mathrm{L}$-alanine by the terminal $\mathrm{C}_{\mathrm{T}}$ domain. ${ }^{11}$ Our initial hypothesis of oxidation of the free $\alpha-\mathrm{NH}_{2}$ of 13 to yield 14 prior to cyclization (path b) as a means to prevent formation of the ten-membered ring $\mathbf{1 0}$ and the subsequent quinazoline product $\mathbf{1 5}$ (path c) is not in play, as no trace of either $\mathbf{1 1}$ or $\mathbf{1 5}$ was detected in the $\Delta p s y C$ strain. Therefore, the active site of PsyB $\mathrm{C}_{\mathrm{T}}$ domain must not only position the indole ring within bond-forming distance from the thioester carbonyl, but also be able to deprotonate the indole $\mathrm{NH}$ proton to initiate the attack. Phylogenetic analysis of the PsyB $C_{T}$ domain with canonical macrocyclizing $\mathrm{C}_{\mathrm{T}}$ domains shows no clear distinction at the protein sequence level. Structural information of the domain will hence provide more insight into its unique catalytic mechanism. Following release of $\mathbf{9}$, the P450 PsyC is able to oxidize the $\operatorname{Trp} \alpha-\mathrm{NH}_{2}$ to the nitro group and yield 2 . We attempted reconstitution of PsyC activities in yeast or using yeast microsomes. However, we were not successful as the failure to reconstitute fungal P450 activities in heterologous hosts is frequently encountered. Nevertheless, it is expected that a similar pathway leads to the formation of the free amine precursors in the biosynthesis of 5-8, followed by $N$-acetylation and methylation to yield the isolated natural products.

Comparison of the metabolite extracts from the wild type and the three $\Delta p s y$ deletion strains revealed significant changes to an additional metabolite $\mathbf{1 0}$ with $\mathrm{m} / z 507(\mathrm{M}+\mathrm{H})^{+}$(Supplementary Figure S7). The compound $\mathbf{1 0}$ was present in all of the strains except $\Delta p s y B$, and was purified from $4 \mathrm{~L}$ culture of the wild type strain. The ${ }^{1} \mathrm{H}$ spectrum of 10 displayed signals of four mono-substituted aromatic rings in the aromatic region and two sets of coupling systems $(\delta 2.87$ (dd, 13.8, 8.4, H-7a), $\delta 2.93$ (dd, 13.8, 6.7, H-7b), $\delta 4.56(\mathrm{~m}, \mathrm{H}-8), \delta 4.40(\mathrm{dd}, J=11.3,4.3 \mathrm{~Hz}, \mathrm{H}-9 \mathrm{a})$ and $\delta 4.13$ (dd, $J=11.3,6.3 \mathrm{~Hz}, \mathrm{H}-9 \mathrm{~b})$ ) and $(\delta 3.29$ (m, H-7'a), $\delta 3.12$ (dd, 13.8, 9.3, H-7'b) and $\left.\delta 4.79\left(\mathrm{~m}, \mathrm{H}-8^{\prime}\right)\right)$. By comparing the ${ }^{1} \mathrm{H}$ and ${ }^{13} \mathrm{C}$ spectra to those reported, ${ }^{12,13} \mathbf{1 0}$ was elucidated as N(N'-benzoyl-Lphenylalaninyl)-L-phenylalaniol benzoate as shown in Figure 1 (Supplementary Table S5). 10 consists of a diphenylalanine backbone that is $N$-acylated with benzoate. The C-terminus of the dipeptide is reduced to an alcohol, and is esterified with another molecule of benzoate. 10 was previously isolated from plant tissues, including the roots of Zeyhera digitalis ${ }^{12}$ and the stems of Celastrus rugosus. ${ }^{13}$

It is very surprising that inactivation of PsyB led to abolishment of 10 production in $P$. rivulum. We propose that PsyB may be involved in the activation of benzoate and loading onto its PCP domain. Previous substrate specificities studies with Ant-activating domains from fungi showed that while Ant is the most optimal substrate, benzoate displayed a $\sim 20 \%$ activity in the ATP-PPi exchange assay. ${ }^{9}$ This indicates that under physiological conditions, benzoate can be loaded on the PsyB if the A domain behaves similarly. Since there in no nucleophilic amine group to attack the dipeptide 12, the benzoyl thioester can instead be attacked by nucleophiles such as amines and alcohols. We suggest the diphenylalanine may be synthesized by a separate NRPS that can reductively offload the peptide with a C-terminal hydroxyl group. Additional catalytic mechanisms may facilitate formation of the benzyl amide and ester present in 10. In this hypothesis, either Ant or benzoate can be activated by PsyB and lead to formation of $\mathbf{2}$ or $\mathbf{1 0}$, respectively. While there is no direct biochemical evidence to support the hypothesis and entirely different biochemical logic may govern the biosynthesis of 10 , our model nevertheless provides an intriguing possibility that a single NRPS is involved in two separate biosynthetic pathways, which may in turn explain why PsyB is found as a standalone NRPS instead of being fused with PsyA in a single polypeptide as in all other fungal NRPS assembly lines found to date.

In conclusion, we identified the biosynthetic pathway of psychrophilin from Penicillium and confirmed the timing of the nitro group formation. The most unusual aspect of the enzymology is the regioselectivity displayed by the $\mathrm{C}_{\mathrm{T}}$ domain of the monomodule NRPS, which warrants additional structural and mechanistic investigations.

\section{CONFLICT OF INTEREST}

The authors declare no conflict of interest.

\section{ACKNOWLEDGEMENTS}

This work is support by the David and Lucile Packard Fellowship in Science and Engineering to YT We thank Leibniz Hang for help with HRMS analysis. This work is supported by NIGMS 5DP1GM106413. We dedicate this submission to Prof. David Cane, who has provided instrumental help to the career of Yi Tang, ever since his postdoctoral stay in Chaitan Khosla's lab.

1 Dalsgaard, P. W., Blunt, J. W., Munro, M. H., Larsen, T. O. \& Christophersen, C. Psychrophilin B and C: cyclic nitropeptides from the psychrotolerant fungus Penicillium rivulum. J. Nat. Prod. 67, 1950-1952 (2004).

2 Dalsgaard, P. W., Larsen, T. O., Frydenvang, K. \& Christophersen, C. Psychrophilin A and cycloaspeptide $D$, novel cyclic peptides from the psychrotolerant fungus Penicillium ribeum. J. Nat. Prod. 67, 878-881 (2004).

3 Ebada, S. S. et al. Psychrophilin E, a new cyclotripeptide, from co-fermentation of two marine alga-derived fungi of the genus Aspergillus. Nat. Prod. Res. 28, 776-781 (2014).

4 Peng, J. et al. Psychrophilins E-H and versicotide C, cyclic peptides from the marinederived fungus Aspergillus versicolor ZLN-60. J. Nat. Prod. 77, 2218-2223 (2014).

5 Walsh, C. T., Haynes, S. W., Ames, B. D., Gao, X. \& Tang, Y. Short pathways to complexity generation: fungal peptidyl alkaloid multicyclic scaffolds from anthranilate building blocks. ACS Chem. Biol. 8, 1366-1382 (2013).

6 Gao, X. et al. Fungal indole alkaloid biosynthesis: genetic and biochemical investigation of the tryptoquialanine pathway in Penicillium aethiopicum. J. Am. Chem. Soc. 133, 2729-2741 (2011).

7 Gao, X. et al. Cyclization of fungal nonribosomal peptides by a terminal condensationlike domain. Nat. Chem. Biol. 8, 823-830 (2012).

8 Medema, M. H. et al. antiSMASH: rapid identification, annotation and analysis of secondary metabolite biosynthesis gene clusters in bacterial and fungal genome sequences. Nucleic Acids Res. 39, W339-W346 (2011).

9 Ames, B. D. \& Walsh, C. T. Anthranilate-activating modules from fungal nonribosomal peptide assembly lines. Biochemistry 49, 3351-3365 (2010).

10 Alexander, N. J., Hohn, T. M. \& McCormick, S. P. The TRI11 gene of Fusarium sporotrichioides encodes a cytochrome P-450 monooxygenase required for C-15 hydroxylation in trichothecene biosynthesis. Appl. Environ. Microbiol. 64 221-225 (1998).

11 Ames, B. D., Liu, X. \& Walsh, C. T. Enzymatic processing of fumiquinazoline F: a tandem oxidative-acylation strategy for the generation of multicyclic scaffolds in fungal indole alkaloid biosynthesis. Biochemistry 49, 8564-8576 (2010).

12 Ferreira, D. T., Silva, R. B., Deoliveira, A. B., Isobe, M. \& Braz Filho, R. Dipeptide from the Roots of Zeyhera-Digitalis. J. Braz. Chem. Soc. 6, 323-326 (1995).

13 Chang, R. J. et al. Chemical constituents of the stems of Celastrus rugosus. Arch. Pharm. Res. 36, 1291-1301 (2013). 